\title{
Memory changes with normal aging: Behavioral and electrophysiological measures
}

\author{
CARRIE A. JOYCE, ${ }^{a}$ KEN A. PALLER, ${ }^{b}$ HEATHER K. MCISAAC, ${ }^{c}$ AND MARTA KUTAS ${ }^{\mathrm{a}, \mathrm{d}}$ \\ ${ }^{a}$ Department of Cognitive Science, University of California, San Diego, USA \\ ${ }^{\mathrm{b}}$ Department of Psychology, Northwestern University, Evanston, IL, USA \\ ${ }^{c}$ Department of Psychology, University of British Columbia, Vancouver, Canada \\ ${ }^{\mathrm{d}}$ Department of Neurosciences, University of California, San Diego, USA
}

\begin{abstract}
We examined performance in young and elderly on an implicit (lexical decision) and an explicit (recognition) memory test. The difference in lexical decision times between old and new words was equivalent in the two groups, although the elderly were slower. In both groups, recognition accuracy (lower in the elderly) was higher following semantic than nonsemantic encoding, whereas lexical decision times were unaffected. Divergent brain potentials for old and new words during lexical decisions constituted a repetition effect, which reflected greater positivity (200-800 ms) for old words, particularly over the left hemisphere; this effect was smaller and later in the elderly. An electrophysiological marker of enhanced recollection for words from the semantic encoding task took the form of a left-sided positivity (500-800 ms). The effect was smaller in the elderly than the young, providing an additional index of their impaired recognition processes.
\end{abstract}

Descriptors: Memory, Aging, ERP, Lexical decision, Levels of processing

Memory loss is one of the most common complaints among the elderly. Yet, delineating the various causes of age-related memory deficits has proven remarkably difficult. Prime among the complicating factors is the discovery that the memory abilities of older adults vary with what memories are queried and how memory is assessed. In general, memory can be probed in two different ways, implicitly and explicitly. Explicit tests, such as recognition and recall, make direct reference to the fact that an item was previously encountered. Implicit tests assess memory by measuring the effects of past experience with an item on subsequent behavior without reference to prior encounters with the item. Differential performance on implicit and explicit tests of memory by patients with brain damage has led to the suggestion that the tests are subserved by nonidentical neural systems (Gabrieli, Fleischman, Keane, Reminger, \& Morrell, 1995; Squire, 1992).

One generalization about memory decline with normal aging has been that elderly individuals, like amnesic patients, exhibit a disproportionate impairment on explicit relative to implicit memory tests. Indeed, younger adults often show a significant advantage over older adults on several explicit measures of memory (Bowles \& Poon, 1981; Chiarello \& Hoyer, 1988; Graf, 1990; Java

This work was supported by research grants from NIMH (52893) and NIA (AG08313), and a graduate fellowship to C.A. Joyce from the McDonnell-Pew Center for Cognitive Neuroscience in San Diego.

Address reprint requests to: Marta Kutas, Department of Cognitive Science, 9500 Gilman Dr., University of California, San Diego, San Diego, CA 92093-0515, USA. E-mail: mkutas@ucsd.edu.
\& Gardiner, 1991; Mitchell, 1989). Age-related effects on implicit tests are less consistent and appear to be more task dependent. For example, when implicit memory is measured in word identification paradigms, young and elderly individuals do not differ (Graf, 1990; Light \& Singh, 1987), whereas in stem completion and word association paradigms, elderly adults report fewer completions from among the studied words, showing less priming than younger adults (Chiarello \& Hoyer, 1988). However, Graf (1990) pointed out that in studies reporting reduced priming effects in the elderly, the reduction was proportionally less than that observed on explicit memory test performance.

Event-related potential (ERP) measures have also been used to investigate memory and aging. Most studies in younger adults have focused on explicit memory tests. The main finding has been an enhanced posterior positivity between 250 and $700 \mathrm{~ms}$ in the ERPs for old compared with new items (Rugg \& Doyle, 1992; Smith \& Halgren, 1989). This ERP repetition effect is presumed to subsume the N400 component and the late positive component (LPC) (Swick \& Knight, 1997). Typically, with repetition N400 amplitude is reduced and LPC amplitude is increased (e.g., Domalski, Smith, \& Halgren, 1991; Smith, Stapleton, \& Halgren, 1986; see Rugg, 1995, for review). Moreover, the LPC is assumed to contain overlapping contributions from the P300 (related to target detection and decision-making) and other potentials specifically related to repetition and retrieval (e.g. Swick \& Knight, 1997; Van Petten \& Rheinfelder, 1995).

Similar ERP effects have been observed in a variety of tasks probing memory implicitly. For example, several investigators have 
found that repeated items (words, nonwords, pictures, or faces) compared with unrepeated items are associated with a larger positive potential between 300 and $600 \mathrm{~ms}$ over centroparietal scalp sites, whether repetition is task-relevant or incidental (Bentin \& McCarthy, 1994; Joyce, Paller, McIsaac, \& Kutas, 1996; Paller, Kutas, \& McIsaac, 1995; Rugg, 1990; Rugg \& Doyle, 1992; Rugg, Furda, \& Lorist, 1988; Rugg \& Nagy, 1987, 1989; Smith \& Halgren, 1987, 1989; Smith et al., 1986). This ERP repetition effect is enhanced by multiple presentations of the same item (Bentin, Moscovitch, \& Heth, 1992) and modulated by levels of processing manipulations (Friedman, Hamberger, \& Ritter, 1993; Hamberger \& Friedman, 1992; Rugg et al., 1988).

Because, under some circumstances, ERP repetition effects have been found to vary with manipulations that affect performance on explicit but not implicit memory tests, Paller and Kutas (1992) suggested that a large portion of them may index recollection processes during the implicit tests (see also Wilding, Doyle, \& Rugg, 1995; Wilding \& Rugg, 1996). In contrast, a small portion of the ERP repetition effect was shown to be modulated by manipulations that affect performance on implicit but not explicit measures of memory (Paller \& Gross, 1998; Paller, Kutas, \& McIsaac, 1998). Swick and Knight (1997) also proposed that modulations in the early, or N400, portions ( $\sim 300-500 \mathrm{~ms})$ of the ERP repetition effect are related to priming, whereas those in the later, or LPC, portions $(\sim 500-800 \mathrm{~ms})$ are related to recollection.

In ERP studies requiring responses to intermittent targets, repeated words universally yield larger positivities than novel words regardless of the age of the individual, although the effect typically starts later in the elderly (Friedman et al., 1993; Friedman, Hamberger, Stern, \& Marder, 1992; Hamberger \& Friedman, 1992; Rugg, Pearl, Walker, Roberts, \& Holdstock, 1994). There is less of a consensus on whether or not the size of the ERP repetition effect is influenced by normal aging. Some researchers have reported enhanced repetition effects in the elderly and attributed them to more extensive processing of repeated items (Friedman et al., 1992, 1993), whereas others have observed no effect of age (Rugg et al., 1994). There are also variable results as to whether ERP repetition effects change in duration with advancing age even when the same task is used (Friedman et al., 1993; Rugg et al., 1994).

These inconsistencies in the data from elderly subjects may be due to differential engagements by various tasks of "priming" and "recollection" processes. In the current study, we systematically manipulated a variable known to influence explicit memory in order to ascertain if reductions in behavioral recognition due to aging are reflected in the portion of the ERP repetition effect thought to index recollection. Specifically, we adopted the studytest design used by Paller et al. (1995), in which semantic versus nonsemantic processing at study resulted in higher recognition accuracy but did not affect lexical decision priming. In that study, ERP amplitudes during the lexical decision task (LDT) were not only more positive for studied relative to new words starting at 300 ms, but also more positive for words studied in the semantic than in the nonsemantic task. This differential ERP repetition effect was interpreted as a sign of recollection.

In the present study, we compared young and elderly groups on these memory and ERP measures. Based on the literature, we expected that the younger group would recognize more words than the elderly with associated ERP reductions primarily in the explicit portions of the repetition effect. We also anticipated that the repetition effect would have a later onset in the elderly given the general slowing of processing and response with advanced aging. In short, we sought to determine the extent to which normal aging differentially influences performance on implicit and explicit memory tests, and on corresponding ERPs.

\section{Methods}

\section{Participants}

Two groups of 20 native English-speaking adults were paid for taking part in a single, 3-hour experimental session. One group consisted of 10 women and 10 men, aged 18-26 years (mean = 20.8), of whom 18 were right-handed and 6 had left-handed members in their immediate family (the data from 11 of the 20 were reported by Paller et al., 1995). The other group consisted of 9 women and 11 men between $60-79$ years of age (mean $=70.95)$ recruited from adult classes, of whom all were right-handed but 11 had left-handed immediate family members. All seniors resided in independent living conditions, transported themselves to the testing site, and were free of major illnesses.

\section{Stimuli}

The stimuli and procedure were identical to those used in Paller et al. (1995, Experiment 2). There were three lists of 150 words each. The assignment of these three lists to the three conditions (semantic study, nonsemantic study, and unstudied) was counterbalanced across subjects. Each list was balanced for word frequency (Kucera \& Francis, 1967) and word length. Overall there were 165 low frequency words (less than 7 occurrences/million), 141 medium frequency words (7-24 occurrences/million), and 144 high frequency words (more than 24 occurrences/million). These included 192 five-letter words, 144 six-letter words, and 114 sevenand eight-letter words. One hundred pseudowords were also used; 50 were presented only once and 50 were presented twice within their respective test blocks. All pseudowords were orthographically plausible. An additional 239 words of comparable length and frequency were used as distractors during the recognition test.

\section{Procedure}

Participants were told that the goal of the experiment was to monitor their brainwaves while they read words and created visual images of the words in their minds. Following electrode application, each participant was taken into a sound-attenuating chamber and seated in a recliner chair approximately $65 \mathrm{~cm}$ from the video monitor. They were instructed to minimize body movements and blinks. Each experimental task was taught separately with several practice trials. For the study and test phases, a white rectangle $\left(4.2^{\circ} \times 1.2^{\circ}\right)$ appeared in the center of the computer monitor. Words were presented inside the rectangle in upper-case letters (vertical visual angle $=0.6^{\circ}$ ). The white rectangle appeared 3,000 $\mathrm{ms}$ prior to the first word of each study and test block. Cues above the white rectangle reminded participants which hand to use for which response and which judgment to make (i.e., small or large size, only 1 or 2 or more syllables, nonword or word, new or old). All responses were given via a button press with one or the other hand.

Participants were given 10 experimental blocks, each consisting of a study phase and a test phase. During the study phase, participants performed both imagery and syllable tasks. In the imagery task, participants were asked to visualize the object represented by the word and indicate whether the object was larger or smaller than the video monitor. In the syllable task, participants were asked to decide whether a given word had one or more than one syllable. In each study block, 15 words were studied in the 
image task and 15 were studied in the syllable task. The two tasks were alternated every other word and task order was counterbalanced across participants. Words were presented for a duration of $300 \mathrm{~ms}$ at a rate of one every $3,000 \mathrm{~ms}$.

Each study phase was followed immediately by a modified LDT in which the 30 words from the preceding study phase were intermixed with 15 new words and 15 pseudowords. Participants were asked to indicate as quickly as possible via button press whether the letter string, presented for $300 \mathrm{~ms}$, was a word or a pseudoword. Two seconds after word onset, the cues above the rectangle signaled the subject to indicate whether or not the item had been seen in the previous study phase.

The experiment concluded with a paper and pencil recognition test in which participants were asked to circle words they remembered seeing during the experiment. This recognition test consisted of 539 words printed in 11 columns on a single sheet of paper.

\section{Electrophysiological Recordings}

Tin electrodes embedded in an elastic cap were used to make recordings from 13 scalp locations of the International 10-20 System (Jasper, 1958): lateral and midline frontal, central and parietal, and lateral temporal and occipital. The reference electrode was the left mastoid, but recordings were re-referenced offline to averaged left and right mastoids. Eye movements and blinks were monitored by electrodes placed near the outer canthi of each eye and beneath the right eye. Trials contaminated by artifacts were eliminated prior to averaging; such trials accounted for approximately $10 \%$ of the trials in young and $15 \%$ in elderly participants. Blinks were identified on a subject-by-subject basis by an algorithm that checked for amplitude of the potential from an electrode below the eye together with polarity inversion between this electrode and a site on the forehead. Electrical activity was amplified with a bandpass of $0.01-100 \mathrm{~Hz}$ and digitized at a rate of $250 \mathrm{~Hz}$. ERPs were computed for epochs extending from $100 \mathrm{~ms}$ prior to word onset to $924 \mathrm{~ms}$ after word onset. Only ERPs recorded during the LDT were analyzed and reported.

\section{Statistical Analyses}

For reaction time data, means and standard deviations for each level of the examined factors were calculated over all correctresponse trials. Analyses of variance (ANOVAs) were conducted on two levels of age group (young, elderly), three levels of study task (words studied under the image task, the syllable task, or new words), and three levels of word frequency (low, medium, high).

For electrophysiological data, the mean amplitudes of various time intervals were calculated relative to the amplitude of the EEG activity in the $100 \mathrm{~ms}$ interval prior to word onset. These data were submitted to a between-subjects ANOVA with two levels of age group (young, elderly), three levels of study task (image, syllable, new), five levels of anterior to posterior electrode sites (frontal, central, parietal, temporal, occipital), and two levels of laterality (left, right). Two-way ANOVAs were conducted to test several a priori hypotheses concerning relations among the image, syllable, and new words conditions. To assess interactions with electrode site, data were normalized across electrodes (McCarthy \& Woods, 1985) prior to conducting ANOVAs. Post hoc analyses were performed using the Tukey test. Reported $F$ ratios and significance values are based on degrees of freedom determined by applying the Huynh-Feldt correction procedure for controlling Type I errors in repeated measures designs.

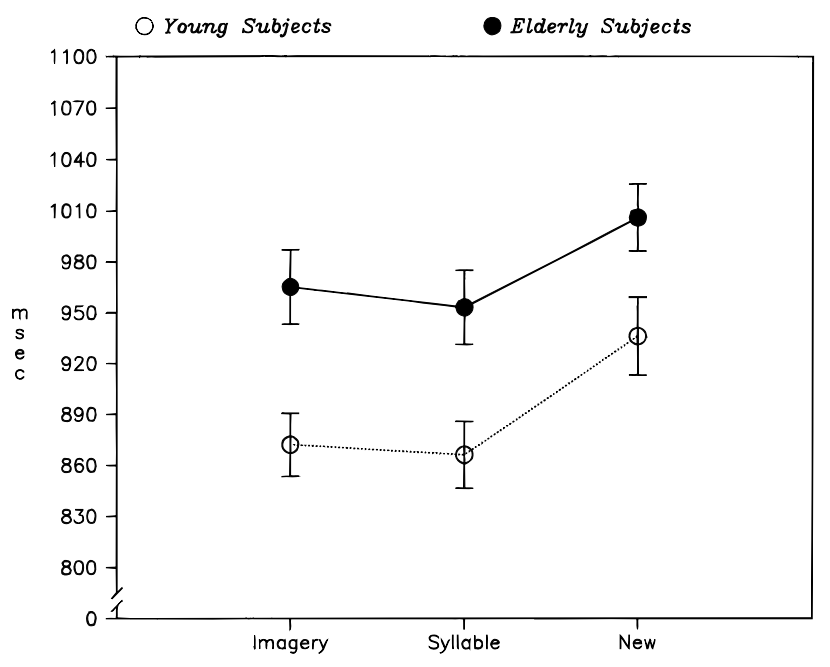

Figure 1. Mean reaction times for young (open circles/dotted lines) and elderly subjects (closed circles/solid line) for lexical decisions to words studied under the imagery and syllable conditions and to unstudied words. Error bars indicate standard error of the mean.

\section{Results}

\section{Lexical Decision Performance}

The mean reaction times for young and elderly participants, collapsed across word frequency, are shown in Figure $1 .{ }^{1}$ The reaction times of the elderly group were significantly slower than those of the young group, $F(1,38)=6.44, p<.02$. The significant main effect of study task, $F(2,76)=43.66, p<.001, \epsilon=0.82$, reflected significantly faster reaction times for studied versus new words (imagery $\sim 40 \mathrm{~ms}$ faster than new, $F(1,38)=41.87, p<.001$; syllable $\sim 44 \mathrm{~ms}$ faster than new, $F(1,38)=68.39, p<.001$. There was no difference between lexical decision times for syllable and image task words and no interaction of task with age. The main effect of word frequency was significant, $F(2,76)=17.64, p<$ .001. A post hoc Tukey test showed that this effect was due to significantly slower reaction times to low than either medium or high frequency words (low $=902 \mathrm{~ms}$; medium $=878 \mathrm{~ms}$; high $=$ $883 \mathrm{~ms}$ ). Word frequency did not interact with age but did interact with study task, $F(4,152)=2.82, p<.03, \epsilon=0.99$, such that reaction times for low frequency words were significantly slower than those to medium and high frequency words for both the image, Low $\times$ Medium $F(1,39)=22.13, p<.001$, Low $\times$ High $F(1,39)=7.99, p<.008$, and new word conditions, Low $\times$ Medium $F(1,39)=40.47, p<.001$, Low $\times$ High $F(1,39)=$ $22.91, p<.001$. There were no frequency differences for words in the syllable condition. Responses to the first and second presentations of pseudowords were not different, nor was there an interaction of pseudoword repetition with age.

\section{Recognition Performance}

Collapsed across test delay, young participants recognized significantly more words than did the elderly, $80 \%$ versus $67 \%$, $F(1,38)=14.94, p<.001$ (see Figure 2). Accuracy for words

\footnotetext{
${ }^{1}$ Analyses were also conducted using trimmed reaction time data (excluding outliers beyond 2.5 SDs from mean) and log transformed reaction times. These analyses yielded identical trends to those of the untrimmed, raw reaction time data.
} 


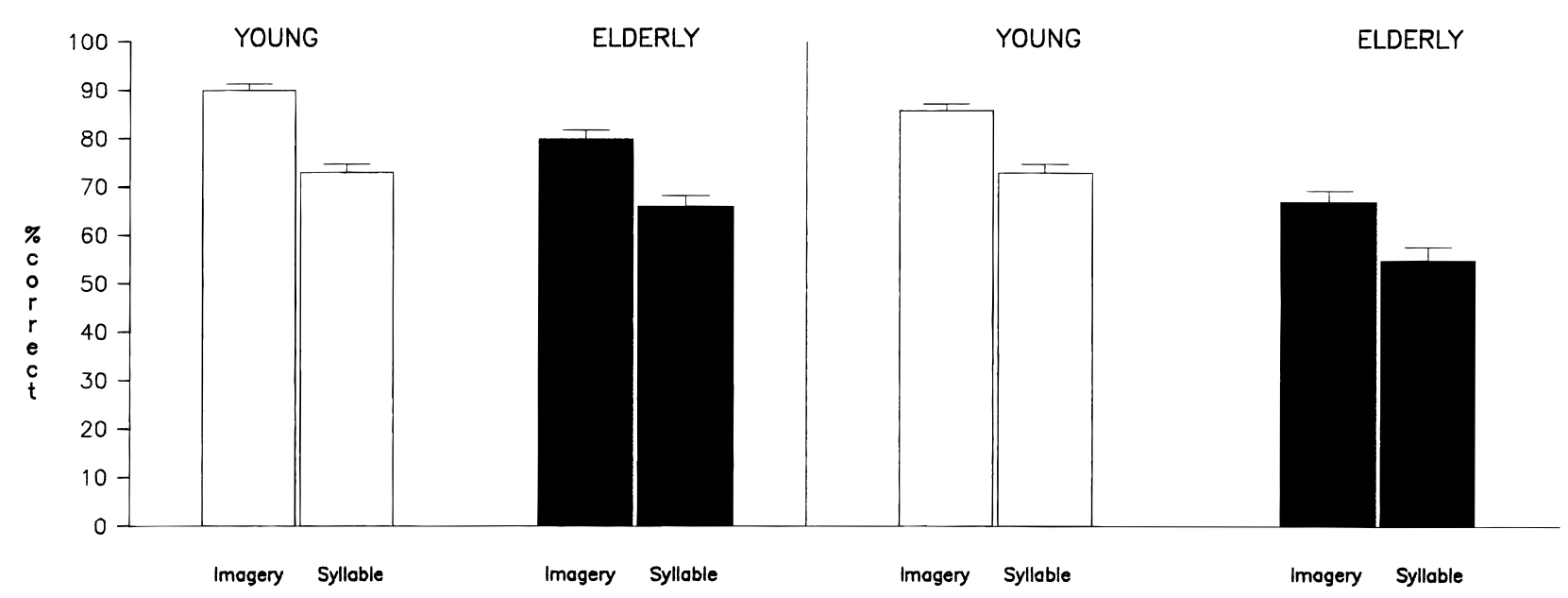

Figure 2. Mean percent correct during the immediate and delayed recognition tests for young (white bars) and elderly (black bars) groups. Error bars indicate standard error of the mean.

from the image task was higher than for words from the syllable task, $81 \%$ versus $67 \%, F(1,38)=135.08, p<.0001$; this effect did not interact with age. A main effect of word frequency, $F(2,76)=35.5, p<.0001, \epsilon=0.94$, reflected better recognition for low $(76 \%)$ than high $(70 \%)$ frequency words, $F(1,38)=4.49$, $p<.05$. Word frequency did not interact either with age or study task. Overall, participants recognized more words in the immediate $(77 \%)$ than subsequent $(70 \%)$ recognition test, $F(1,38)=9.25$, $p<.005$. Test delay interacted marginally with age, $F(1,38)=$ $3.99, p=.053$, reflecting a larger age-related difference on the second recognition test. Signal detection analyses showed that in the elderly group, the bias to respond "yes" was stronger in the first recognition test (immediate: $d$-prime $=1.57$, beta $=1.31$; delayed: $\mathrm{d}$-prime $=1.86$, beta $=3.32$ ). In both groups, significantly more false alarms were committed in the immediate $(14 \%)$ than in the delayed $(6 \%)$ tests $F(1,38)=20.90, p<.0001$; this effect was larger in elderly participants (immediate: $17 \%$, delayed: $6 \%$ ) than in younger participants (immediate: $11 \%$, delayed: $7 \%$ ), age by test delay: $F(1,38)=5.00, p<.04$. Recognition test delay did not interact with study task or word frequency.

\section{Electrophysiological Results}

ERP results are shown in Figure 3 for midline sites and in Figure 4 for lateral sites. During the test phase, ERPs to words for both young and elderly participants exhibited a small N1 $(\sim 80 \mathrm{~ms})$, followed by P2 ( $200 \mathrm{~ms})$, N400 (300-500 ms) and LPCs ( 500$800 \mathrm{~ms}$ ). The N400 was smaller and the P300 at posterior sites less pronounced in the elderly than in the younger participants. The onset latencies of the P2, N400, and P300 components were approximately the same for both groups, although the repetition effect began later in the older group.

ERP data were quantified by measuring mean amplitudes to capture early (200-500 ms) and late (500-800 ms) repetition effects (e.g., Swick \& Knight, 1997). The distribution of these two measures across the scalp are plotted in Figure 5 separately for each age group, study task, and electrode. As there were no significant interactions of study task with either word frequency or word length in either age group, these two variables were omitted from further analyses. All $F$ values reported below pertaining to interactions with electrode were obtained on normalized data.

Between 200-500 ms, there was a significant main effect of age, $F(1,38)=5.32, p<.03$, reflecting larger $\mathrm{P} 2$ and smaller N400 amplitudes in the elderly (see Figure 3 ). There was also a significant main effect of study task, $F(2,76)=16.14, p<.001$, $\epsilon=0.78$; mean amplitudes were more positive for words studied under the imagery and syllable tasks than for new words, $F(1,38)=19.30, p<.001$ and $F(1,38)=17.78, p<.001$, respectively. Words from the imagery task elicited significantly larger positivities than those from the syllable task, $F(1,38)=7.03, p<$ .02 . There was also a Study task $\times$ Age interaction, $F(2,76)=$ $5.65, p<.006, \epsilon=0.78$, reflecting significant study effects in the young, imagery vs. new $F(1,19)=16.58, p<.001$; syllable vs. new $F(1,19)=15.46, p<.001$, but not in the elderly participants.

An Age $\times$ Electrode interaction, $F(4,152)=14.65, p<.001$, $\epsilon=0.48$, reflected greater frontal positivity in the elderly than in the young for all words; the younger participants showed the largest positivity over parietal scalp sites (see Figures 4 and 5). A marginal interaction of study task with anterior/posterior electrode distribution was mainly due to syllable task words eliciting more positive potentials than new words over posterior sites, $F(4,152)=$ 3.63, $p<.07, \epsilon=0.36$. A significant interaction of task with laterality was due to larger differences between imagery and new words over the left than right hemisphere, $F(1,38)=6.32, p<.02$. Analyses of image minus new and syllable minus new difference waves revealed a larger study task (i.e., repetition priming) effect over left hemisphere sites in the young than in the elderly for words from the image but not the syllable condition, $F(1,38)=$ $5.15, p<.03$ (Figure 6).

Between 500-800 ms, there was no main effect of age nor any significant interactions of age with study task. There was, however, a main effect of study task, $F(2,76)=12.23, p<.001, \epsilon=0.78$, reflecting the greater positivity elicited by image words relative to both syllable, $F(1,38)=33.70, p<.001$, and new words, $F(1,38)=15.54, p<.001$. ERPs to syllable and new words did not differ significantly from each other. There were significant interactions of age and electrode site revealing that (1) the re- 
YOUNG

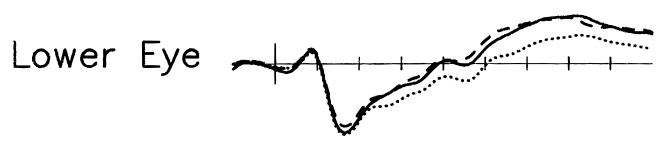

Frontal

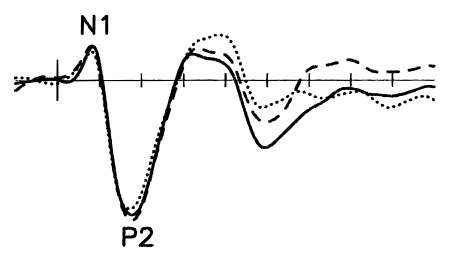

Central

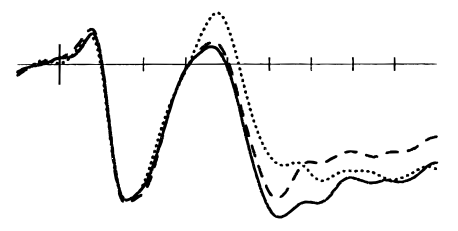

Parietal
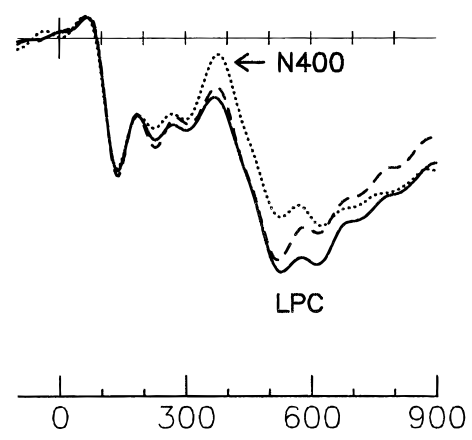

ELDERLY
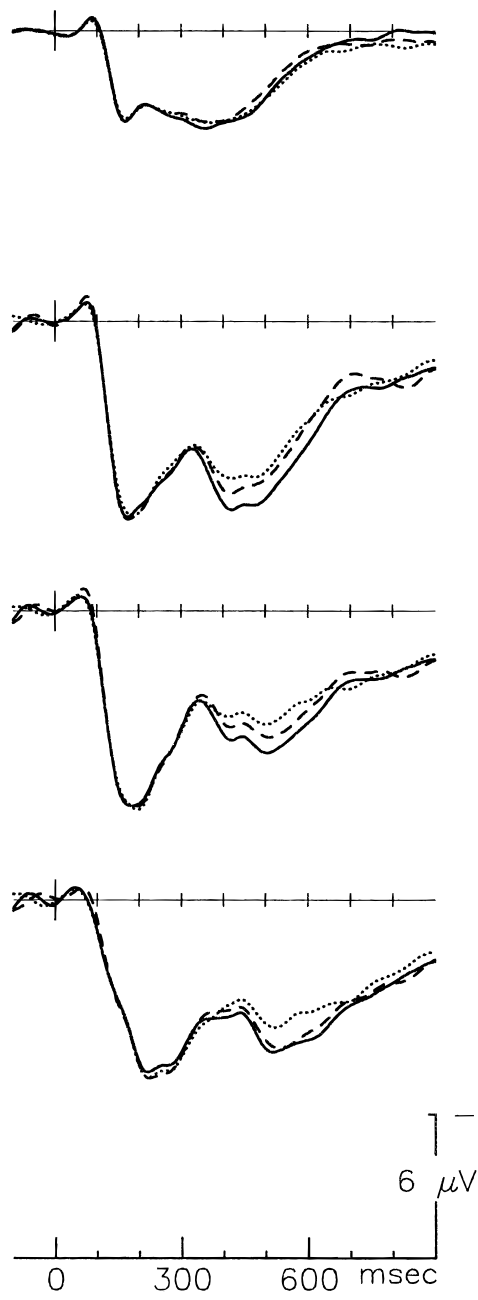

New

Figure 3. Event-related potentials (ERPs) from midline electrodes recorded during the lexical decision test for young (left column) and elderly (right column) participants.

sponses of the elderly were characterized by relatively greater frontal positivity than those of the young, although at all other sites the ERPs of the young showed greater positivity, Age $\times$ Anterior/ posterior distribution, $F(4,152)=6.81, p<.002, \epsilon=0.50$, see Figures 4 and 5; (2) in the elderly, this late positivity was slightly larger over right than left hemisphere frontocentral sites, ${ }^{2}$ whereas for both groups it was larger over left than right hemisphere posterior sites, Age $\times$ Anterior/posterior $\times$ Laterality $F(4,152)=$ 4.44, $p<.015, \epsilon=0.54$, see Figures 4 and 5. Analyses of the image minus new and syllable minus new difference waves re-

${ }^{2}$ Whereas overall the elderly group exhibited more eye movements than the younger adults, regression analyses indicated that the right frontal laterality in the elderly was not due to the influence of horizontal eye movements $(r=.01)$. vealed significant Age $\times$ Laterality interactions for image minus new difference waves, $F(1,38)=8.01, p<.007$, verifying that young and elderly differed primarily in the presence of a larger late positivity in the image condition over the left hemisphere in younger subjects (see bottom of Figure 6).

\section{Discussion}

As expected, our elderly participants were not as accurate as the younger participants in deciding whether or not a word had also been presented earlier (about $3 \mathrm{~min}$ ) in the experiment. Specifically, they failed to recognize as many old words as the young adults and exhibited a greater tendency to falsely identify words new to the experiment as old. In both age groups, semantic encoding (imagery task) led to better recognition memory perfor- 
YOUNG
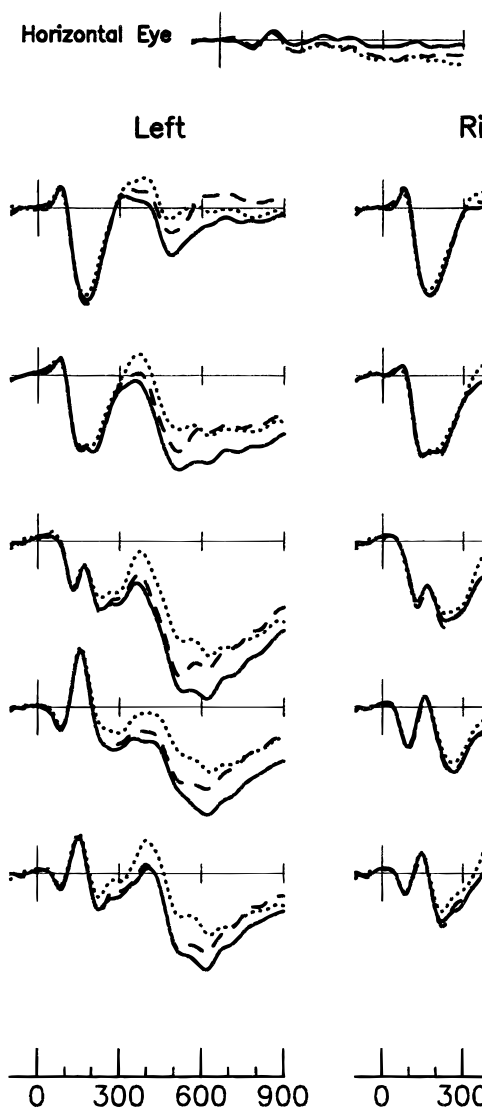

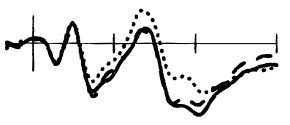

Right
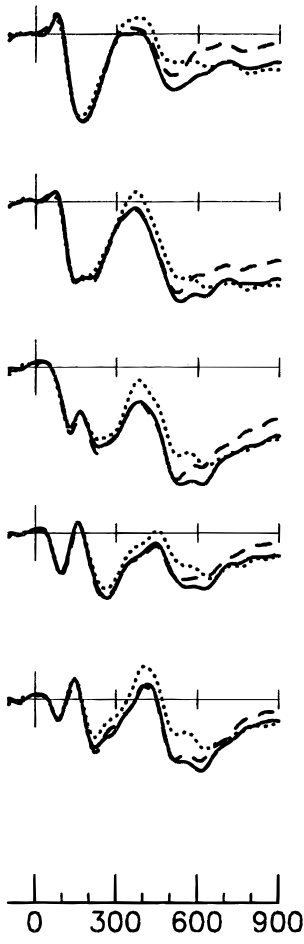

\section{ELDERLY}

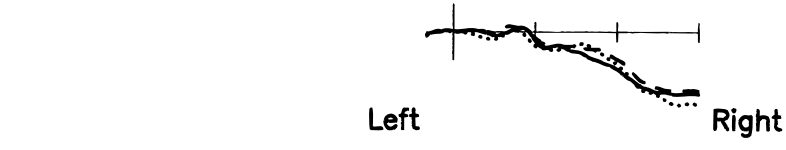

Frontal

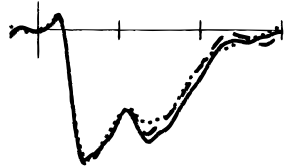

Central

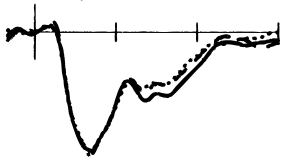

Parietal

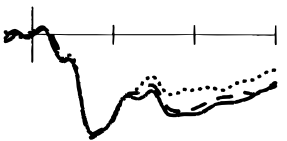

Temporal
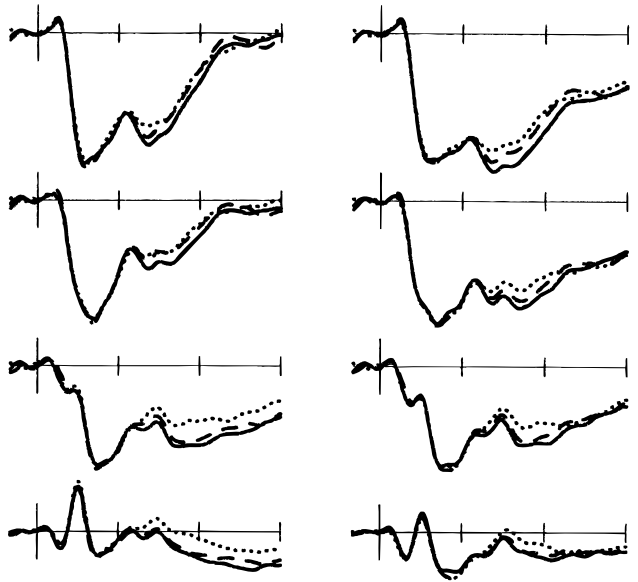

Occipital
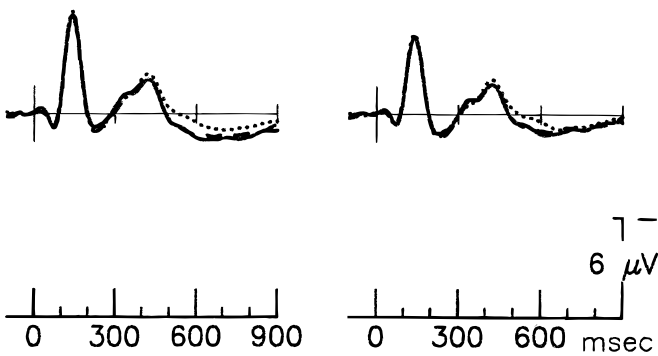

New

Figure 4. Event-related potentials (ERPs) from lateral electrodes elicited by old words studied in the imagery and syllable conditions and new words during the LDT in the young (left) and elderly (right) participants. ERPs are shown from electrode sites over frontal, central, parietal, temporal, and occipital regions of the left (left column) and right (right column) hemispheres.

mance than did orthographic encoding (syllable task), consistent with the levels-of-processing framework (e.g., Jacoby \& Dallas, 1981). In addition, although lexical decision times to studied words were faster than those to words occurring for the first time in the experiment, the times were unaffected by the levels of processing manipulation. In other words, we observed the typical repetition priming effect on lexical decision times and replicated its insensitivity to semantic processing.

We also found that ERPs recorded during the LDT showed a repetition effect, which, unlike lexical decision times, was sensitive to the levels of processing manipulation. That is, (1) ERPs to studied words were generally more positive than those to new words from $300 \mathrm{~ms}$ on and (2) this positivity was larger for words from the imagery than the syllable study task. This pattern of results was also observed by Paller et al. (1995), and following their argument, we take this differential ERP repetition effect (larger positivity to image than syllable words) to be a reflection of recollection during the implicit memory task.

The pattern of each of the memory-related effects was remarkably similar for elderly and young adults. Both groups showed (1) better recognition for words from the imagery than the syllable condition; (2) faster lexical decision times to studied than to new words; (3) no reliable difference in lexical decision times for old words from the imagery and syllable study tasks; (4) greater late positivity for studied than for new words; and (5) greater late positivity for image than for syllable studied words. These results as a whole suggest that highly similar mechanisms determined memory performance in older and younger adults.

The primary differences between the younger and older adults were in overall recognition accuracy, response speed, ERP waveshape and distribution, and the influence of study task on the ERP repetition effect. As has been previously reported by others (Bowles \& Poon, 1981; Friedman et al., 1992, 1993), lexical decision times were slower and the onset of the ERP repetition effect was later in the elderly participants ${ }^{3}$. At the same time, the morphology and

${ }^{3}$ Although the ERP repetition effect is thought to be composed of multiple overlapping components, such as the N400, LPC, and perhaps a $\mathrm{P} 3 \mathrm{~b}$, discussing the repetition effects in the current data in terms of these subcomponents does nothing to further our understanding of either the components or the repetition effect. The current data cannot elucidate differential contributions of these components. We thus treat the ERP repetition effect in the present experiment as a unitary phenomenon, although we do measure the first and second halves separately. 
YOUNG

A. $200-500 \mathrm{~ms}$

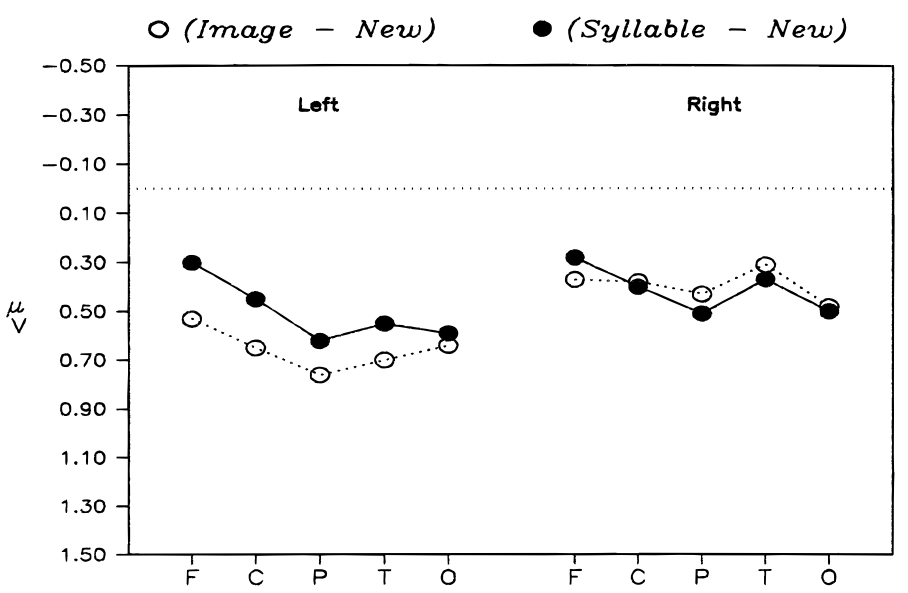

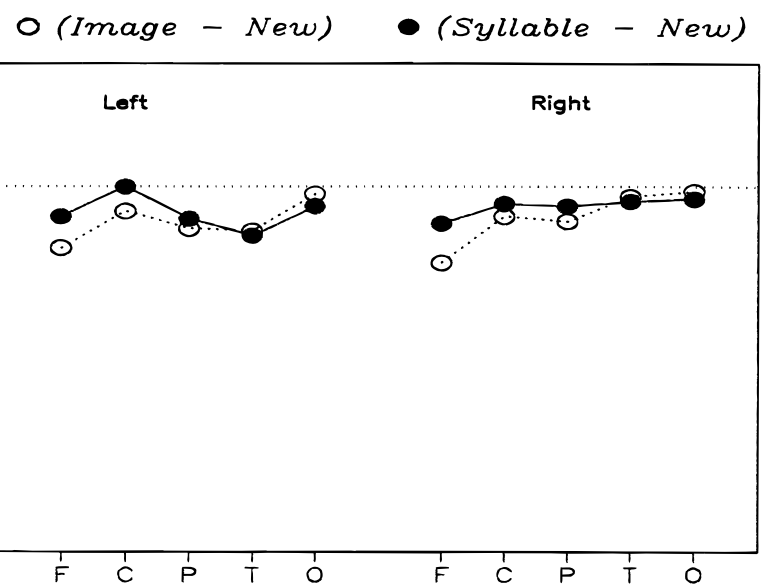

\section{B. $500-800 \mathrm{~ms}$}
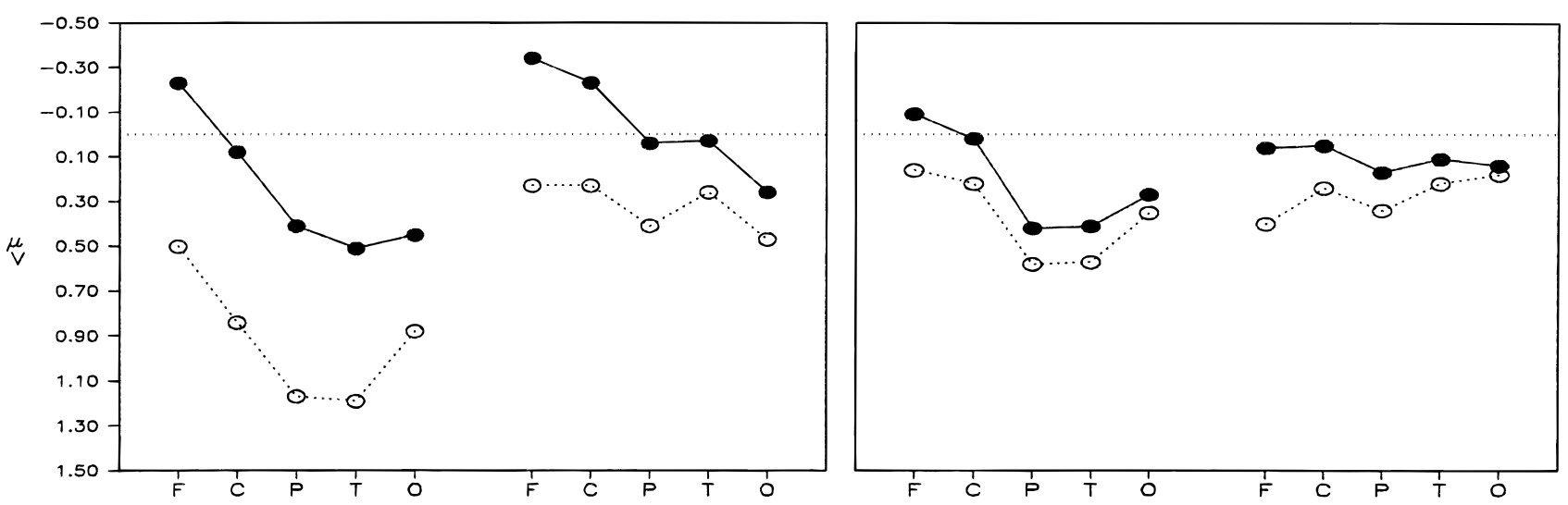

Figure 5. Comparison of the distributions of event-related potential (ERP) mean amplitudes for the younger (dashed line) and older (solid line) groups. Shown separately are comparisons for words studied in the imagery (top panel) and syllable (middle panel) conditions, and new words (bottom panel) and for the 200-500- (left) and 500-800-ms (right) intervals.

scalp distribution of the ERPs to words along both the anterior/ posterior and left/right dimensions were affected by aging. For example, the later portion of the ERP repetition effect from 500 to $800 \mathrm{~ms}$ was left-dominant in the younger group, especially for semantically studied words, but bilaterally symmetric for the elderly group (see bottom of Figure 6). In addition, whereas ERPs during the LDT in both groups were characterized by a late positivity between 300 and $900 \mathrm{~ms}$, scalp distribution of this positivity varied. In the younger adults, this late positivity had the typical P3b distribution, largest parietally and decreasing toward the front of the head. In the older adults, this late positivity was more frontally distributed. We, along with other researchers, have observed this age-related frontal predominance in P3b amplitude to nonverbal stimuli in both the auditory and visual modalities (De Jong, Kok, Woestenburg, Logman, \& Van Rooy, 1988; Friedman et al., 1993; Iragui, Kutas, Mitchiner, \& Hillyard, 1993; Kutas, Iragui, \& Hillyard, 1994; Nielsen-Bohlman \& Knight, 1995; Pfefferbaum, Ford, Roth, \& Kopell, 1980; Pfefferbaum, Ford, Wenegrat, Roth, \& Kopell, 1984; Smith, Michalewski, Brent, \& Thompson, 1980; Swick \& Knight, 1997; but see Picton, Stuss, Champagne, \& Nelson, 1984, for an exception).
Several hypotheses have been offered to account for these various patterns of topographical changes in the P3b with age. Some accounts attribute the apparent change in P3b distribution to an overlap with another ERP component sensitive to normal aging, such as a central positive wave (Picton et al., 1984) or the frontal slow wave (Pfefferbaum et al., 1984). Swick and Knight (1997) suggested that the greater frontal positivity in the elderly in their studies may reflect increases in effort or sustained attention for the elderly to perform at a high level. Whatever the explanation, none of the available evidence suggests that this particular age-related change in P3b topography is specific to memory, although there may be indirect effects on memory (e.g., through strategic choices).

In contrast, we believe that the age-related changes in the lateral asymmetry of the late positivity in the present experiment are, at least in part, memory-related. Specifically, the late positivity (500-800 ms) over the back of the head in the younger adults is larger over left than right hemisphere sites, particularly for studied words. By contrast, the frontal late positivity $(500-800 \mathrm{~ms})$ in the elderly participants is larger over the right than the left hemisphere and only affected by repetition of semantically studied words (i.e., not different for syllable vs. new words from 500 to $800 \mathrm{~ms}$ ). We 

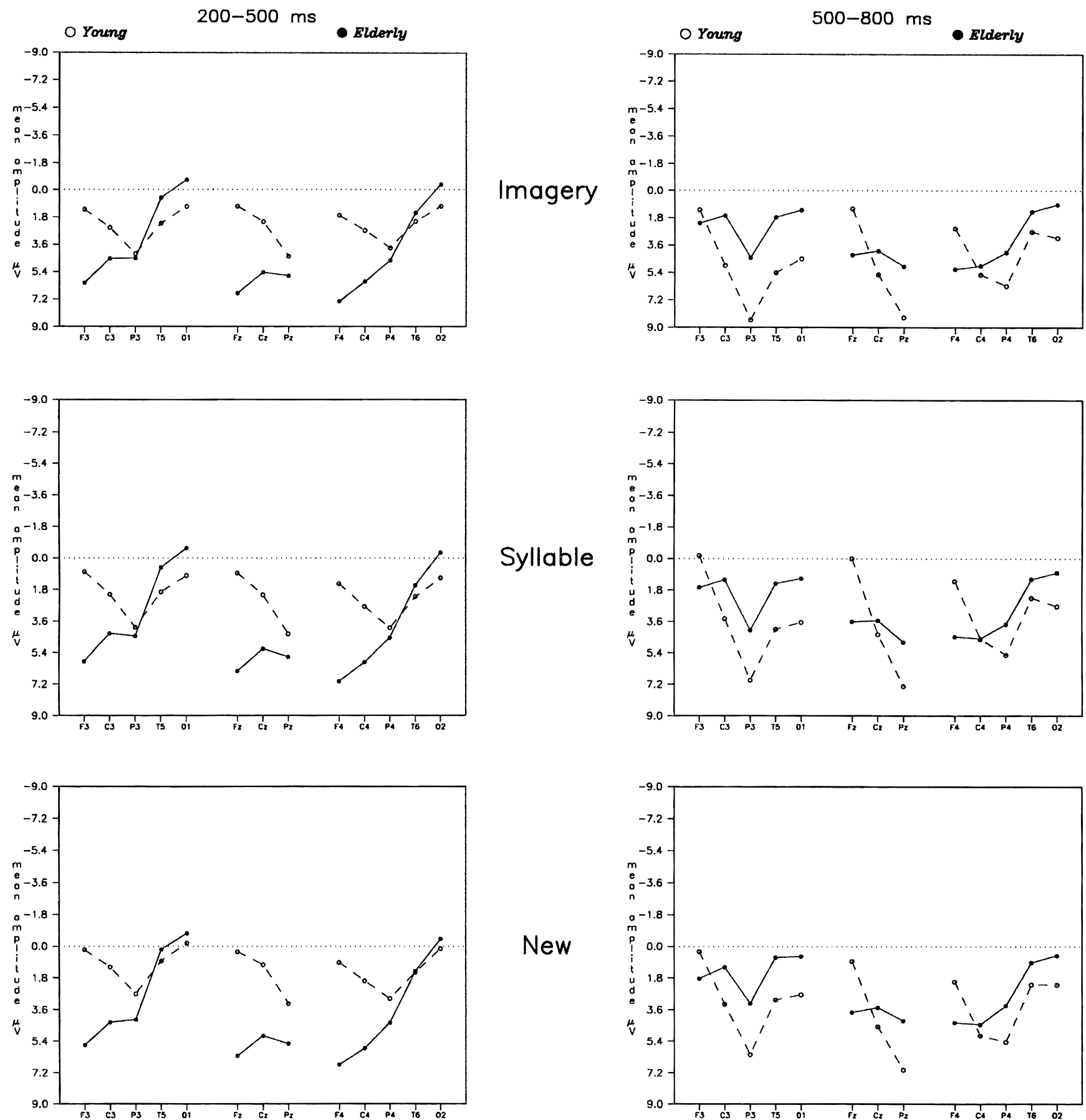

Figure 6. Mean amplitudes of the difference event-related potentials (ERPs) during the 200-500- (top) and 500-800-ms (bottom) intervals for young (left) and elderly (right) participants during the lexical decision task. Frontal, central, parietal, temporal, and occipital sites are presented for both hemispheres. Shown superimposed are the difference ERPs obtained by subtracting responses to new words from responses to words studied in the imagery (solid line) and syllable (dotted line) conditions.

suggest that this pattern of differential laterality reflects the greater involvement of recollective processes in young than in elderly adults and is consistent with the conclusion that normal aging impacts explicit more than implicit memory processes. This theory follows from our hypothesis that the differential ERP repetition effect between image and syllable words is a sign of recollection. Likewise, other ERP studies of recognition have shown larger ERP repetition effects in conditions associated with more extensive recollective processing (Wilding et al., 1995; Wilding \& Rugg, 1996).
Generally speaking, ERP repetition effects in implicit and explicit memory paradigms resemble each other (e.g., Swick \& Knight, 1997). The timing of these ERP repetition effects, which onset around $300 \mathrm{~ms}$, is suggestive of an association with explicit memory processes. Based on the view that implicit memory reflects changes in sensory and perceptual processes, effects on implicit memory might be expected to occur somewhat earlier. And, as has been noted by others (Haist \& Kutas, submitted; Paller \& Gross, 1998), some effects of priming are seen on the early sensory com- 
ponents such as the $\mathrm{N} 1$ and $\mathrm{P} 2$ (less than $250 \mathrm{~ms}$ ). Its timing aside, whereas this ERP repetition effect is observed during both implicit and explicit tests of memory, the effect is typically larger and more robust in explicit tests. Moreover, the size of this ERP repetition effect is influenced by experimental manipulations (levels of processing) that are known to influence explicit and generally not implicit measures of memory. In our experiments, words from the image condition were not only recognized more accurately than those from the syllable condition, but also associated with a larger repetition effect in both the young and the elderly. Also consistent with this view is the observation that the ERP repetition effect was larger when recollection processes were accentuated by interleaving lexical decision and recognition judgments than when only lexical decisions were required (Paller et al., 1995).

In their direct comparison of ERPs in an LDT and recognition memory task, Swick and Knight (1997) found, in line with our hypothesis, that the repetition effects were larger when memory was tested explicitly in their younger subjects. In their older subjects the repetition effects were larger in the implicit memory test. They suggested that some of these effects were influenced by the subject's confidence in his or her judgments, which they presumed to be greater for the elderly in the easier LDT than in the recognition memory task. Their elderly participants differed most from the younger participants in the recognition task, for which they not only exhibited smaller but also later and more abbreviated ERP repetition effects, especially at the longest repetition lags. Swick and Knight interpreted the findings as indicating that, whereas explicit memory declines with age, implicit memory remains largely intact.

We, too, found that the ERP repetition effect was smaller, later, and somewhat shorter in duration among the elderly participants, although not to the extent found by Swick and Knight (1997). Several methodological differences between the present experiment and that of Swick and Knight might account for this apparent discrepancy. First, ours was not a continuous LDT but was a studytest design with a longer delay between study and test. Further, we presented two study tasks and our most obvious group differences were seen in those words studied semantically. Moreover, we increased the likelihood of explicit processes during the LDT by interleaving the recognition judgments. Undoubtedly, this too had the effect of making the LDT more difficult, as was evident in the relatively slow lexical decision times in both age groups.

As noted above, the largest group differences in the ERP repetition effect were for words that had been deeply encoded (imaged) and thus were most likely to be remembered. In the younger adults, the late positivity (P3b) elicited by image task words was especially large over the left hemisphere. This asymmetric part of the late positivity was much reduced for words whose first occurrence had been in the syllable count task and virtually absent for new (unstudied) words. The asymmetry was absent from the ERPs to any words in the elderly group. It is this part of the ERP repetition effect, the differential amplitude and distribution between image and syllable studied words, that we believe to be an index of the differential recollection of these words. It is possible that the reduction in the elderly of this component may have been a result of their devoting more energy to the lexical decisions than to the recognition judgments during the interleaved portion of the test. However, the fact that the elderly showed parallel behavioral deficits in the interleaved and delayed recognition tests supports the recollection interpretation.

This ERP lateralization in the young adults is consistent with data from other neuroimaging techniques. For example, an fMRI study by Demb et al. (1995) showed decreased activation in the left inferior prefrontal cortex (LIPC) for repeated words that had been studied semantically but not nonsemantically. They concluded that LIPC activation is related to semantic encoding, which is known to lead to better recognition. This has also been suggested by positron emission tomography (PET) studies, which have noted differential left prefrontal activation for semantic versus nonsemantic encoding conditions (Kapur, Craik, 1994; Kapur, Rose, 1994). Further, Demb et al. (1995) suggested that the decrease they observed in the LIPC only to semantically encoded, repeated words reflected a process-specific facilitation of retrieval. Under this view, the lack of lateralization in our elderly group may reflect their less efficient and/or less elaborate semantic processing (relative to younger adults) rather than a problem with memory storage and retrieval per se.

Another possibility is that the left-lateralized ERP effects are related to successful retrieval. For instance, Nyberg, McIntosh, Houle, Nilsson, and Tulving (1996) observed higher PET activation in the left medial temporal lobe for recognition following semantic than perceptual encoding; furthermore, the level of activation in the anterior portions of the left parahippocampal gyrus varied with the number of words recognized. This finding suggests a correlation with retrieval success. Thus, perhaps the left laterality of the repetition effect in younger subjects actually reflects the more successful retrieval following semantic encoding.

In summary, our ERP data suggest that memory-related changes in normal aging occur primarily in explicit memory and in relation to semantic processing more than nonsemantic processing. Whether this difference is due to changes in encoding, storage, or retrieval processes is as yet undetermined. Generally lower recognition scores in the elderly relative to the young adults, regardless of how words were studied, suggest an additional role for decision making processes in explicit memory performance. Whatever the ultimate causes of the decline in explicit memory performance with normal aging, it is important to note that the change was disproportionately larger than the changes in implicit memory performance. Determining the precise causes of age-related memory changes will require delineating what factors influence explicit memory in general, and how they might change over time.

\section{REFERENCES}

Bentin, S., \& McCarthy, G. (1994). The effects of immediate stimulus repetition on reaction time and event-related potentials in tasks of different complexity. Journal of Experimental Psychology: Learning, Memory, and Cognition, 20, 130-149.

Bentin, S., Moscovitch, M., \& Heth, I. (1992). Memory with and without awareness: Performance and electrophysiological evidence of savings. Journal of Experimental Psychology: Learning, Memory, and Cognition, 18, 1270-1283.

Bowles, N. L., \& Poon, L. W. (1981). The effect of age on speed of lexical access. Experimental Aging Research, 7, 417-425.

Chiarello, C., \& Hoyer, W. J. (1988). Adult age differences in implicit and explicit memory: Time course and encoding effects. Psychology and Aging, 3, 358-366.

De Jong, H. L., Kok, A., Woestenburg, J. C., Logman, C. J., \& Van Rooy, J. C. G. M. (1988). Learning where to look: Electrophysiological and behavioral indices of visual search in young and old subjects. Biological Psychology, 26, 277-298.

Demb, J. B., Desmond, J. E., Wagner, A. D., Vaidya, C. J., Glover, G. H., \& Gabrieli, J. D. E. (1995). Semantic encoding and retrieval in the left inferior prefrontal cortex: A functional MRI study of task difficulty and process specificity. Journal of Neuroscience, 15, 5870-8. 
Domalski, P., Smith, M. E., \& Halgren, E. (1991). Cross-modal repetition effects on the N4. Psychological Science, 2, 173-8.

Friedman, D., Hamberger, M., \& Ritter, W. (1993). Event-related potentials as indicators of repetition priming in young and older adults: Amplitude, duration, and scalp distribution. Psychology and Aging, 8, 120125 .

Friedman, D., Hamberger, M., Stern, Y., \& Marder, K. (1992). Eventrelated potentials (ERPs) during repetition priming in Alzheimer's patients and young and older controls. Journal of Clinical and Experimental Neuropsychology, 14, 448-462.

Gabrieli, J. D. E., Fleischman, D. A., Keane, M. M., Reminger, S. L., \& Morrell, F. (1995). Double dissociation between memory systems underlying explicit and implicit memory in the human brain. Psychological Science, 6, 76-82.

Graf, P. (1990). Life-span changes in implicit and explicit memory. Bulletin of the Psychonomic Society, 28, 353-358.

Haist, F., \& Kutas, M. (1997). Evidence for the functional independence of priming and declarative memory: Cognitive electrophysiological studies of visual word stem completion. Manuscript submitted for publication.

Hamberger, M., \& Friedman, D. (1992). Event-related potential correlates of repetition priming and stimulus classification in young, middle-aged, and older adults. Journal of Gerontology, 47, 395-405.

Iragui, V. J., Kutas, M., Mitchiner, M. R., \& Hillyard, S. A. (1993). Effects of aging on event-related brain potentials and reaction times in an auditory oddball task. Psychophysiology, 30, 10-22.

Jacoby, L. L., \& Dallas, M. (1981). On the relationship between autobiographical memory and perceptual learning. Journal of Experimental Psychology: General, 110, 306-340.

Jasper, H. H. (1958). The 10-20 system of the International Federation. Electroencephalography and Clinical Neurophysiology, 10, 371-375.

Java, R. I., \& Gardiner, J. M. (1991). Priming and aging: Further evidence of preserved memory function. American Journal of Psychology, 104, 89-100.

Joyce, C. A., Paller, K. A., McIsaac, H. K., \& Kutas, M. (1996, March). You can say THET again: ERP repetition effects on nonwords. Poster presented at the meeting of the Cognitive Neuroscience Society, San Francisco, CA.

Kapur, S., Craik, F. I., Tulving, E., Wilson, A. A., Houle, S., \& Brown, G. M. (1994). Neuroanatomical correlates of encoding in episodic memory: Levels of processing effect. Proceedings of the National Academy of Sciences, USA, 91, 2008-11.

Kapur, S., Rose, R., Liddle, P. F., Zipursky, R. B., Brown, G. M., Stuss, D., Houle, S., \& Tulving, E. (1994). The role of the left prefrontal cortex in verbal processing: Semantic processing or willed action? NeuroReport, 5, 2193-6.

Kucera, H., \& Francis, W. N. (1967). Computational analysis of presentday American English. Providence, RI: Brown University Press.

Kutas, M., Iragui, V., \& Hillyard, S. A. (1994). Effects of aging on eventrelated brain potentials (ERPs) in a visual detection task. Electroencephalography and Clinical Neurophysiology: Evoked Potentials, 92, 126139.

Light, L. L., \& Singh, A. (1987). Implicit and explicit memory in young and older adults. Journal of Experimental Psychology: Learning, Memory, and Cognition, 13, 531-541.

McCarthy, G., \& Wood, C. C. (1985). Scalp distributions of event-related potentials: An ambiguity associated with analysis of variance models. Electroencephalography and Clinical Neurophysiology, 62, 203-8.

Mitchell, D. B. (1989). How many memory systems? Evidence from aging. Journal of Experimental Psychology: Learning, Memory, and Cognition, 15, 31-49.

Nielsen-Bohlman, L., \& Knight, R. T. (1995). Prefrontal alterations during memory processing in aging. Cerebral Cortex, 5, 541-549.

Nyberg, L., McIntosh, A. R., Houle, S., Nilsson, L. G., \& Tulving, E. (1996). Activation of medial temporal structures during episodic memory retrieval. Nature, 380, 715-7.

Paller, K. A., \& Gross, M. (1998). Brain potentials associated with perceptual priming versus explicit remembering during the repetition of visual word-form. Neuropsychologia, 36, 559-571.

Paller, K. A., \& Kutas, M. (1992). Brain potentials during memory retrieval provide neurophysiological support for the distinction between conscious recollection and priming. Journal of Cognitive Neuroscience, 4 , 375-391.

Paller, K. A., Kutas, M., \& McIsaac, H. K. (1995). Monitoring conscious recollection via the electrical activity of the brain. Psychological Science, 6, 107-111.

Paller, K. A., Kutas, M., \& McIsaac, H. K. (1998). An electrophysiological measure of priming of visual word form. Consciousness and Cognition, 7, 54-66.

Pfefferbaum, A., Ford, J. M., Roth, W. T., \& Kopell, B. S. (1980). Agerelated changes in auditory event-related potentials. Electroencephalography and Clinical Neurophysiology, 49, 266-276.

Pfefferbaum, A., Ford, J. M., Wenegrat, B. G., Roth, W. T., \& Kopell, B. S. (1984). Clinical application of the P3 component of event-related potentials: I. Normal aging. Electroencephalography and Clinical Neurophysiology: Evoked Potentials, 59, 85-103.

Picton, T. W., Stuss, D. T., Champagne, S. C., \& Nelson, R. F. (1984). The effects of age on human event-related potentials. Psychophysiology, 21, 312-325.

Rugg, M. D. (1985). The effects of semantic priming and word repetition on event-related potentials. Psychophysiology, 22, 642-647.

Rugg, M. D. (1990). Event-related brain potentials dissociate repetition effects of high- and low-frequency words. Memory and Cognition, 18, 367-379.

Rugg, M. D. (1995). Event-related potential studies of human memory. In M. S. Gazzaniga (Ed.), The cognitive neurosciences (pp. 789-801) Cambridge, MA: MIT Press.

Rugg, M. D., \& Doyle, M. C. (1992). Event-related potentials and recognition memory for low- and high-frequency words. Journal of Cognitive Neuroscience, 4, 69-79.

Rugg, M. D., Furda, J., \& Lorist, M. (1988). The effects of task on the modulation of event-related potentials by word repetition. Psychophysiology, 25, 55-63.

Rugg, M. D., \& Nagy, M. E. (1987). Lexical contribution to nonwordrepetition effects: Evidence from event-related potentials. Memory and Cognition, 15, 473-481.

Rugg, M. D., \& Nagy, M. E. (1989). Event-related potentials and recognition memory for words. Electroencephalography and Clinical Neurophysiology, 72, 395-406.

Rugg, M. D., Pearl, S., Walker, P., Roberts, R. C., \& Holdstock, J. S. (1994). Word repetition effects on event-related potentials in healthy young and old subjects, and in patients with Alzheimer-type dementia. Neuropsychologia, 32, 381-398.

Smith, D. B., Michalewski, H. J., Brent, G. A., \& Thompson, L. W. (1980) Auditory averaged evoked potentials and aging: Factors of stimulus, task and topography. Biological Psychology, 11, 135-151.

Smith, M. E., \& Halgren, E. (1987). Event-related potentials during lexical decision: Effects of repetition, word frequency, pronounceability, and concreteness. Electroencephalography and Clinical Neurophysiology. 40 (Suppl.), 417-421.

Smith, M. E., \& Halgren, E. (1989). Dissociation of recognition memory components following temporal lobe lesions. Journal of Experimental Psychology: Learning, Memory, and Cognition, 15, 50-60.

Smith, M. E., Stapleton, J. M., \& Halgren, E. (1986). Human medial temporal lobe potentials evoked in memory and language tasks. Electroencephalography and Clinical Neurophysiology, 63, 145-159.

Squire, L. R. (1992). Memory and the hippocampus: A synthesis from findings with rats, monkeys, and humans. Psychological Review, 99, 195-231.

Swick, D., \& Knight, R. T. (1997). Event-related potentials differentiate the effects of aging on word and nonword repetition in explicit and implicit memory tasks. Journal of Experimental Psychology: Learning, Memory, and Cognition, 23, 123-142.

Van Petten, C., \& Rheinfelder, H. (1995). Conceptual relationships between spoken words and environmental sounds: Event-related brain potential measures. Neuropsychologia, 33, 485-508.

Wilding, E. L., Doyle, M. C., \& Rugg, M. D. (1995). Recognition memory with and without retrieval of context: An event-related potential study. Neuropsychologia, 33, 743-767.

Wilding, E. L., \& Rugg, M. D. (1996). An event-related potential study of recognition memory with and without retrieval of source. Brain, 119, 889-905.

(ReceIved January 14, 1997; ACCEPTED December 11, 1997) 